\title{
Precise-Orientation-Beamforming Scheme for Wireless Communications between Buoys
}

\author{
Zhihui Wu, Bin Zhang, Fengzhong Qu, Ye Cheng, Zhujun Zhang, \\ Ying Ye, Ying Chen, and Liuqing Yang
}

Ocean College, Zhejiang University, Hangzhou 310058, China

Correspondence should be addressed to Fengzhong Qu; jimqufz@163.com

Received 27 December 2015; Revised 25 April 2016; Accepted 16 May 2016

Academic Editor: Marco Mussetta

Copyright (C) 2016 Zhihui Wu et al. This is an open access article distributed under the Creative Commons Attribution License, which permits unrestricted use, distribution, and reproduction in any medium, provided the original work is properly cited.

\begin{abstract}
Utilizing wireless sensor network (WSN) to monitor the marine environment is one of the major techniques in oceanographic monitoring, and how to increase the limited communication distance between the buoys in WSN has become a hot research issue. In this paper, a new technique called precise-orientation-beamforming (POB) which uses the beamforming algorithm to increase the communication distance between buoys is presented. As was widely applied in the radar and sonar, the beamforming method was not used to extend the communication distance between buoys so far. The POB method overcomes the unstable position of buoys caused by waves by implementing the orientation filter. The whole process includes two steps: First, the real-time attitude of the antenna array is calculated by the orientation filter. With the known relative direction of the destination node to the antenna array, the second step is to control phased array antenna beamforming parameters, directing the beam at the destination node. The POB scheme has been simulated under the condition of regular waves. The results reveal that POB provides significant power gains and improves the distance between two communicating nodes effectively.
\end{abstract}

\section{Introduction}

The wireless communications for oceanographic monitoring typically include satellite communications [1], commercial cellular network access, for example, 3G [2], and approaches designed for wireless sensor networks (WSNs), for example, ZigBee [2,3]. Satellite communication is a relatively expensive solution with limited capacity and the commercial cellular network only covers offshore areas. WSNs have many advantages in oceanographic monitoring, such as the flexible network topologies and cost-effectiveness in data collection [2]. However, because of the limited communication distance, there need to be a large number of relay buoys deployed in the WSNs, which consist of the major part of the fundamental cost. As a result, researchers are devoted to proposing methods to extend the communication distance between buoys.

Beamforming is a signal processing technique which has found numerous applications in radar, sonar, and wireless sensor networks. The basic idea of beamforming is to direct the majority of signal energy transmitted from the transmit antenna array in a chosen angular direction and effectively extend the communication distance [4] between nodes. For now, beamforming technology has not been widely applied in wireless communications between buoys. The main reason is that the beamforming direction changes as the buoy moves and rotates with the wave. For the current beamforming technology, transmission side requires the channel information $[5,6]$. The channel information is obtained by channel estimation from the receiver and then is fed back to the sender. In condition of long-range and low signal to noise ratio (SNR), channel estimation is not easy and the feedback link from the receiver to sender is hard to establish.

This paper presents a precise-orientation-beamforming (POB) scheme between buoys without channel estimation and feedback from receiver. The investigation is performed by installing an Inertial Movement Unit (IMU) on the buoy to track the rotational and translational movements of the buoy. The real-time attitude of the transmit antenna array, which is fixed on the buoy, is calculated using the orientation filter 


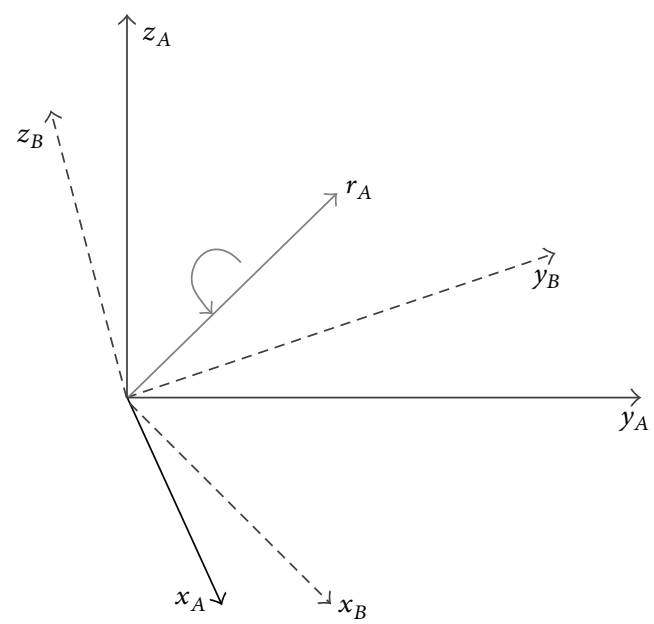

FIgURE 1: The orientation of frame $B$ is achieved by a rotation, from alignment with frame $A$, of angle $\theta$ around the axis $r_{A}$.

[7]. After calculating the real-time posture of the antenna, the relative direction of the target node to the transmit antenna array is gained. As a result, the beam can be steered directly to the target node without channel information. Then a corresponding algorithm [4, 8, 9] is selected to control beamforming parameters of phased array antennas, directing the beam at the target node. To verify the effect of the scheme mentioned above, simulations are performed.

This paper is organized as follows. The measurement of orientation of the antenna array and beamforming method are presented in Section 2. In Section 3, the pattern of the antenna array and the movement of the buoy are both simulated. By tracking the movement of the buoy, beamforming is performed and the power gain is obtained. Finally, summarizing remarks are given in Section 4.

\section{Method}

To apply the beamforming technique to the buoys which move and rotate with waves, the POB scheme first calculates the relative direction of the destination node to the transmitting antenna arrays. After obtaining the relative direction of the target buoy, beamforming is then performed. In this section, the basic theoretical foundations of an orientation filter presented by [7] are first introduced. Then the uniform rectangular array (URA) algorithm is chosen to perform beamforming.

2.1. Measurement of Orientation. A quaternion is a fourdimensional complex number that can be used to represent the orientation of a ridged body or coordinate frame in threedimensional space [7].

An arbitrary orientation of frame $B$ relative to frame $A$ can be achieved through a rotation of angle $\theta$ around an axis $r_{A}$ defined in frame $A$. This is represented graphically in Figure 1, where the mutually orthogonal unit vectors $x_{A}$, $y_{A}$, and $z_{A}$ and $x_{B}, y_{B}$, and $z_{B}$ define the principle axis of coordinate frames $A$ and $B$, respectively. The quaternion describing this orientation, ${ }_{B}^{A} q$, is defined by

$$
\begin{aligned}
{ }_{B}^{A} q & =\left[q_{1}, q_{2}, q_{3}, q_{4}\right] \\
& =\left[\cos \frac{\theta}{2},-r_{x} \sin \frac{\theta}{2},-r_{y} \sin \frac{\theta}{2},-r_{z} \sin \frac{\theta}{2}\right],
\end{aligned}
$$

where $r_{x}, r_{y}$, and $r_{z}$ define the components of the unit vector $r_{A}$ in the $x$-, $y$-, and $z$-axis of frame $A$, respectively.

Euler angles $\psi, \theta$, and $\phi$ describe an orientation of frame $B$ achieved by the sequential rotations, from alignment with frame $A$, of $\psi$ around $z_{A}, \theta$ around $y_{A}$, and $\phi$ around $x_{A} \cdot \psi$ is defined by

$$
\psi=\arctan \frac{2 q_{2} q_{3}-2 q_{1} q_{4}}{2 q_{1}^{2}+2 q_{2}^{2}-1}
$$

$\theta$ is defined by

$$
\theta=-\arcsin \left(2 q_{2} q_{4}+2 q_{1} q_{3}\right)
$$

$\phi$ is defined by

$$
\phi=\arctan \frac{2 q_{3} q_{4}-2 q_{1} q_{2}}{2 q_{1}^{2}+2 q_{4}^{2}-1} .
$$

To ensure the accurate measurement of orientation of the buoy, this paper adopts the orientation filter presented by [7] to track the movement of the buoys. The filter employs a quaternion representation of orientation to describe the real-time posture. In this paper, the filter is directly adopted (as shown in Figure 2) to track the movement of antenna array. The major foundations involved in the filter would be presented and the relative parameters and their significance are shown as follows. follows:

The related parameters of the block diagram are as $a_{t}$ : normalized accelerometer measurement, $s_{\omega_{t}}:$ angular rate vector, ${ }_{E}^{S} \widehat{q}_{\text {est }, t-1}$ : estimate of orientation at time $t-1$, ${ }_{E}^{S} \widehat{q}_{\text {est }, t}:$ estimate of orientation at time $t$,

${ }_{E}^{S} \dot{q}_{\text {est, }, t}$ : the change rate of estimate of orientation at time $t$,

$f_{g}\left({ }_{E}^{S} \widehat{q}_{\text {est }, t-1}, a_{t}\right):$ the objective function,

$J_{g}^{T}\left({ }_{E}^{S} \widehat{q}_{\text {est }, t-1}\right):$ the Jacobian of the objective function,

$\beta$ : the magnitude of the gyroscope measurement error.

An estimated orientation of the sensor frame relative to the earth frame, ${ }_{E}^{S} q_{\text {est }, t}$ is obtained through the fusion of the orientation calculations, ${ }_{E}^{S} q_{w, t}$ and ${ }_{E}^{S} q_{\nabla, t}$, which represent the orientation related to angular rate and vector observations, respectively.

${ }_{E}^{S} q_{w, t}$ is the orientation derived from angular rate which could be obtained from gyroscope. A triaxis gyroscope will 


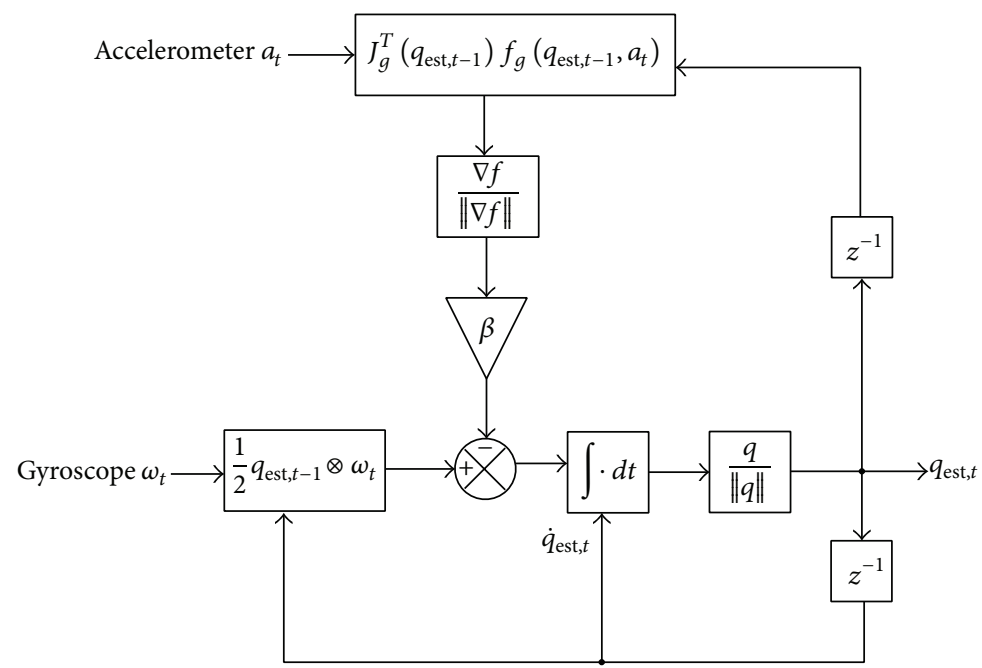

FIGURE 2: Block diagram representation of the complete orientation filter for an IMU implementation.

measure the angular rate about the $x$-, $y$-, and $z$-axis of the senor frame, termed $w_{x}, w_{y}$, and $w_{z}$, respectively. These parameters measured at time $t$ are represented in quaternion and form the angular rate vector $w_{t}$. As the initial conditions are known, the rate of change of orientation of the earth frame relative to the sensor frame ${ }_{E}^{S} \dot{q}_{w, t}$ could be calculated as

$$
{ }_{E}^{S} \dot{q}_{w, t}=\frac{1}{2}{ }_{E}^{S} \widehat{q}_{\mathrm{est}, t-1} \otimes s_{w_{t}} .
$$

The orientation of the earth frame relative to the sensor frame at time $t,{ }_{E}^{S} q_{w, t}$, could be obtained by numerically integrating the quaternion derivative ${ }_{E}^{S} \dot{q}_{w, t}$ :

$$
{ }_{E}^{S} q_{w, t}={ }_{E}^{S} \widehat{q}_{\mathrm{est}, t-1}+{ }_{E}^{S} \dot{q}_{w, t} \Delta t
$$

where ${ }_{E}^{S} q_{\nabla, t}$ is the orientation derived from vector observations. A triaxis accelerometer will measure the magnitude and direction of the field of gravity in the sensor frame compounded with linear accelerations due to motion of the sensor. Equation (7) computes the gradient of the solution surface defined by the objective function and its Jacobian. The subscript $\nabla$ indicates that the quaternion is calculated using the gradient descent algorithm:

$$
\nabla f\left({ }_{E}^{S} \widehat{q}_{k},{ }^{E} \widehat{d},{ }_{\widehat{s}}\right)=J^{T}\left({ }_{E}^{S} \widehat{q}_{k},{ }^{E} \widehat{d}\right) f\left({ }_{E}^{S} \widehat{q}_{k},{ }^{E} \widehat{d},{ }_{\widehat{s}}\right),
$$

where ${ }^{E} \widehat{d}$ is the measured direction of the field in the sensor frame, ${ }^{S} \widehat{s}$, using the rotation operation in [7].

Equation (8) calculates the estimated orientation ${ }_{E}^{S} q_{\nabla, t}$ computed at time $t$ based on a previous estimate of orientation ${ }_{E}^{S} \widehat{q}_{\text {est }, t-1}$ and the objective function gradient $\nabla f$ defined by sensor measurements ${ }^{S} \widehat{a}_{t}$ and ${ }^{S} \widehat{m}_{t}$ sampled at time $t$ :

$$
{ }_{E}^{S} q_{\nabla, t}={ }_{E}^{S} \widehat{q}_{\mathrm{est}, t-1}-\mu_{t} \frac{\nabla f}{\|\nabla f\|} .
$$

An optimal value of $\mu_{t}$ can be defined as that which ensures the convergence rate of ${ }_{E}^{S} q_{\nabla, t}$ is limited to the physical orientation rate as this avoids overshooting due an unnecessarily large step size.

The fusion of ${ }_{E}^{S} q_{w, t}$ and ${ }_{E}^{S} q_{\nabla, t}$ could be calculated by

$$
{ }_{E}^{S} q_{\mathrm{est}, t}=\gamma_{t}{ }_{E}^{S} q_{\nabla, t}+\left(1-\gamma_{t}\right){ }_{E}^{S} q_{w, t}, \quad 0 \leq \gamma_{t} \leq 1 .
$$

An optimal value of $\gamma_{t}$ could be calculated by

$$
\left(1-\gamma_{t}\right) \beta=\gamma_{t} \frac{\mu_{t}}{\Delta t} \text {. }
$$

The orientation filter computes one iteration per time sample provided that the convergence rate governed by $\alpha$ is equal to or greater than the physical rate of change of orientation. The step size is decided by the optimal value $\mu_{t}$.

2.2. Beamforming Implementation. After obtaining the relative direction of the target node to the antenna array, beamforming can be performed to direct the beam at the target node. Several schemes can be used to perform beamforming $[10,11]$. This paper takes the uniform rectangular array (URA) as an example, as shown in Figure 3. Array elements are distributed in the $x z$-plane. $x$ and $z$ are shown in Figure 3, representing the spacing of the adjacent units of $(x, z)$-axis, respectively. When using a digital phase shifter to perform beamforming, the phase value of cell $\left(x_{m}, z_{n}\right)$ relative to cell $(0,0)$ is expressed as

$$
\varphi\left(x_{m}, z_{n}\right)=m \varphi_{x_{0}}+n \varphi_{z_{0}},
$$

where

$$
\begin{aligned}
& \varphi_{x_{0}}=\frac{\left((2 \pi d x / \lambda) \cos \varphi_{x}\right)}{\left(2 \pi / 2^{K}\right)} \\
& \varphi_{z_{0}}=\frac{\left((2 \pi d z / \lambda) \cos \varphi_{z}\right)}{\left(2 \pi / 2^{K}\right)},
\end{aligned}
$$

where $\left(\varphi_{x_{0}}, \varphi_{z_{0}}\right)$ represents the initial vector, $\left(\varphi_{x}, \varphi_{z}\right)$ represent the angle of the beamforming direction and planar array coordinates $(x, z)$-axis, and $K$ represents the number of digital phase shifter bits $[4,8,9]$. 


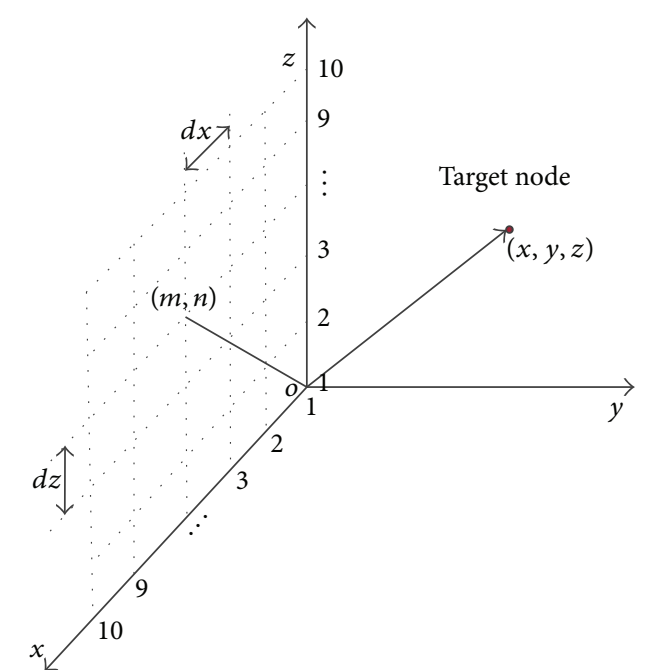

Figure 3: Phased array coordinate schematic diagram.

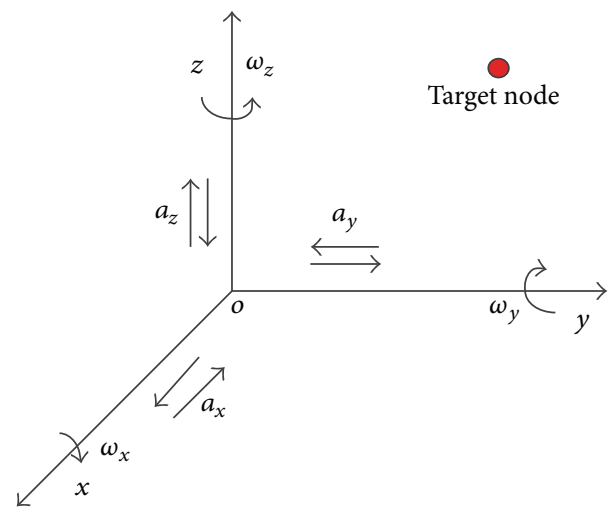

FIGURE 4: Inertial sensor coordinate frame.

\section{Simulation}

In order to verify the effect of the scheme proposed in this paper, simulations are performed under the condition of regular waves. Under regular waves, the movement of the buoy is assumed to be the sway along $y$-axis, the heave along $z$-axis, and the rotation around $x$-axis, as shown in Figure 4. The simulation steps are as follows. First, the movement of the buoy is simulated under the influence of regular waves, as shown in Figure 6, and then the parameters measured by gyroscopes and accelerometers are determined. Secondly, the parameters gained in the previous step are taken as the input to the system, and then the Euler parameters $\psi, \theta$, and $\phi$ are obtained using the oriental filter. Finally, knowing the error of the attitude and the antenna array pattern after beamforming, the power gain over the time is obtained.

3.1. Antenna Array Pattern. This paper takes a $10 \times 10$ URA as an example, as shown in Figure 3. Array elements are distributed in the $x z$-plane. Assume the carrier frequency of the incoming narrow band sources is $300 \mathrm{MHz}$. Use a rectangular lattice, with the default spacing of half wavelength along both the row and column dimensions of the array. Each element is an isotropic antenna element. The antenna array pattern at the direction of any degrees' azimuth and any degrees' elevation is available through simulation. The antenna array pattern at the target direction of 30 degrees' azimuth and 50 degrees' elevation is shown in Figure 5. Figure 5 shows that the biggest normalized power gain after beamforming is at the direction of 30 degrees' azimuth and 50 degrees' elevation.

3.2. The Movement of Buoy under the Condition of Regular Waves. Numerical wave tank (NWT) has been presented for the investigation of hydrodynamic characteristics of floating structures. Compared with traditional physical tests, NWT provides more detailed flow field information with smaller scale effect and lower cost. Wave-generating boundary method that defines the particle velocity and wave height on inlet boundary is introduced in this paper. The $x$-direction velocity is

$$
\begin{aligned}
u= & \frac{\pi H}{T} \frac{\cosh k(y+d)}{\sinh k d} \cos (k x-\omega t) \\
& +\frac{3}{4} \frac{\pi H}{T}\left(\frac{\pi H}{L}\right) \frac{\cosh 2 k(y+d)}{\sinh ^{4} k d} \cos 2(k x-w t),
\end{aligned}
$$

the $y$-direction velocity is

$$
\begin{aligned}
u= & \frac{\pi H}{T} \frac{\cosh k(y+d)}{\sinh k d} \sin (k x-\omega t) \\
& +\frac{3}{4} \frac{\pi H}{T}\left(\frac{\pi H}{L}\right) \frac{\sinh 2 k(y+d)}{\sinh ^{4} k d} \sin 2(k x-w t),
\end{aligned}
$$

and the particle's vertical displacement is

$$
\begin{aligned}
\eta(x, t)=\frac{H}{2} & \cos (k x-\omega t)+\frac{H}{8}\left(\frac{\pi H}{L}\right) \\
& \cdot \frac{\cosh k d}{\sinh ^{3} k d}(\cosh 2 k d+2) \cos 2(k x-\omega t),
\end{aligned}
$$

where $T$ represents wave period, $k$ represents wave number, $H$ represents wave height, $d$ represents water depth, $\omega$ represents circular frequency, $t$ represents time, $x$ represents particle horizontal coordinates, and $y$ represents particle vertical coordinates [12].

This paper simulates the wave using wave-generating boundary method and the Fluent user-defined function. The buoy is assumed to be a floating cylinder. The fluid volume function is adopted to track the free surface of the fluid and the movement of the buoy over the time. The parameters of the regular wave and the buoy are shown in Table 1. Based on the simulation results, the movement of the buoy is presented in Figure 6. As shown in Figure 6, the blue trace represents the vertical displacement $s_{z}$, the red one represents the horizontal displacement $s_{y}$, and the black one represents the angular rotation $\phi$. 


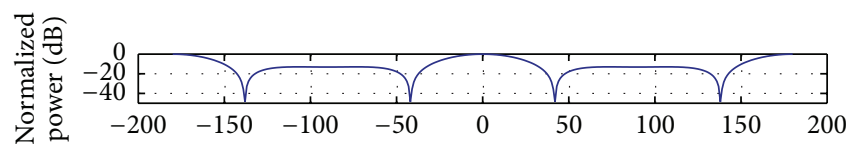

(a) Azimuth angle (degrees)

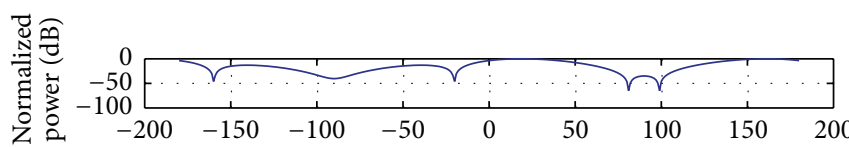

(c) Azimuth angle (degrees)

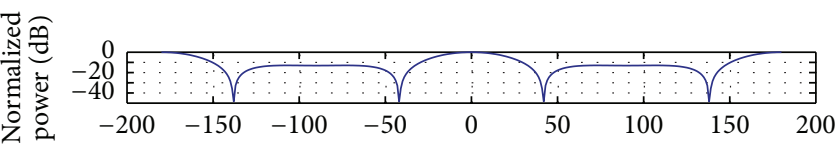

(b) Elevation angle (degrees)

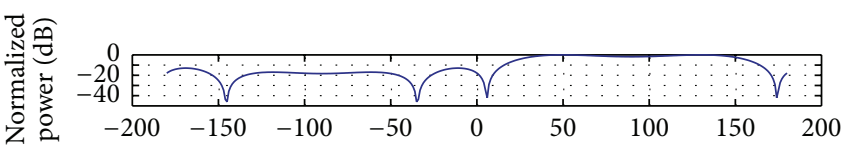

(d) Elevation angle (degrees)

FIGURE 5: The beam pattern before and after beamforming: (a) and (b) are before beamforming and (c) and (d) are after beamforming.

TABLE 1: The parameters of regular wave and buoy.

\begin{tabular}{lc}
\hline Parameters & Magnitude \\
\hline Wave length & $10 \mathrm{~m}$ \\
Wave height & $0.8 \mathrm{~m}$ \\
Acceleration of gravity & $9.81 \mathrm{~m} / \mathrm{s}^{2}$ \\
Depth of water & $3 \mathrm{~m}$ \\
The diameter of floating body & $1 \mathrm{~m}$ \\
The height of floating body & $0.5 \mathrm{~m}$ \\
The density of floating body & $0.5 \mathrm{~kg} / \mathrm{m}^{3}$ \\
\hline
\end{tabular}

3.3. Beamforming. With the movement parameters $s_{z}, s_{y}$, and $\phi$ of the buoy, the system input $a_{t}$ is given by

$$
a_{t}=\left[0, a_{y}, a_{z}\right]^{T}
$$

where

$$
\begin{aligned}
& a_{y}=\frac{d^{2} s_{z}}{d t^{2}} \cdot \sin (\phi)+\frac{d^{2} s_{y}}{d t^{2}} \cdot \cos (\phi)+\frac{d^{2} \phi}{d t^{2}} \cdot \frac{\pi}{180}, \\
& a_{z}=\frac{d^{2} s_{z}}{d t^{2}} \cdot \cos (\phi)-\frac{d^{2} s_{y}}{d t^{2}} \cdot \sin (\phi)-\left(\frac{d \phi}{d t}\right)^{2} \cdot \frac{\pi^{2}}{180^{2}},
\end{aligned}
$$

and $\omega_{t}$ is expressed as

$$
\omega_{t}=\left[\omega_{x}, 0,0\right]^{T},
$$

where

$$
\omega_{x}=\frac{d \phi}{d t}
$$

which are used as the input of the system. Therefore, the real-time attitude is tracked using the filter mentioned above by calculating ${ }_{E}^{S} q_{\text {est }, t}$. The sampling period is assumed to be $2 \mathrm{~ms}$, while the gyroscope measurement error is assumed to be $5 \mathrm{deg} / \mathrm{s}$. Euler parameters $\psi, \theta$, and $\phi$ are computed directly from quaternion data using (2), (3), and (4). Knowing the real-time attitude of the antenna array and the initial position of the two buoys, beamforming is performed using the scheme mentioned above.

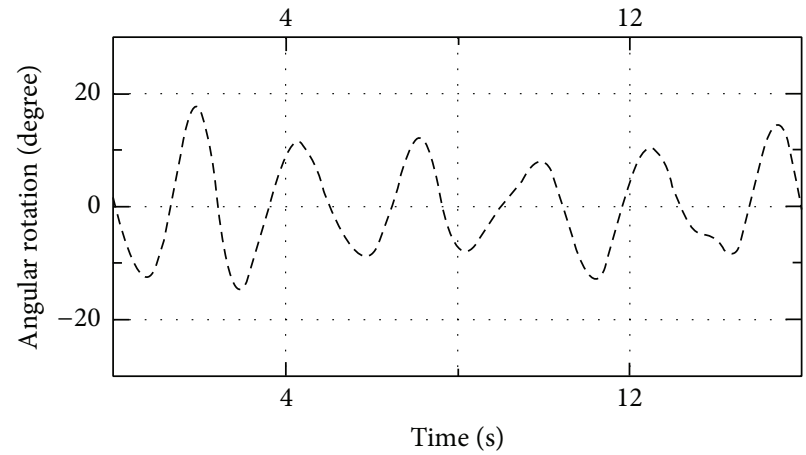

- - Angular rotation

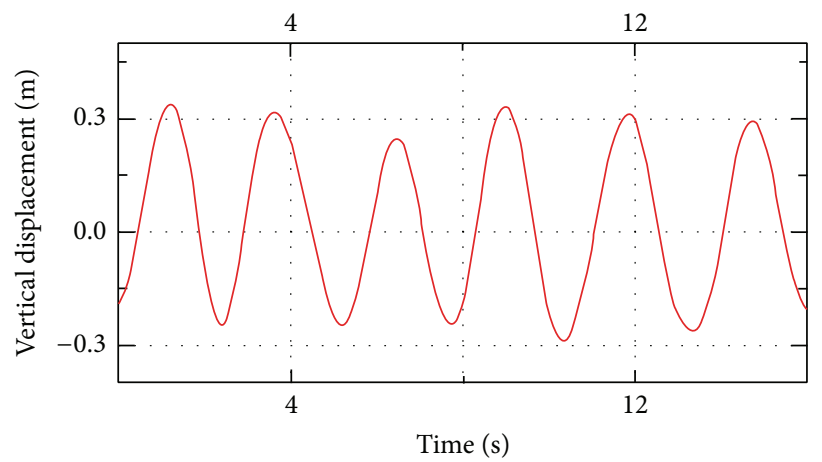

- Vertical displacement

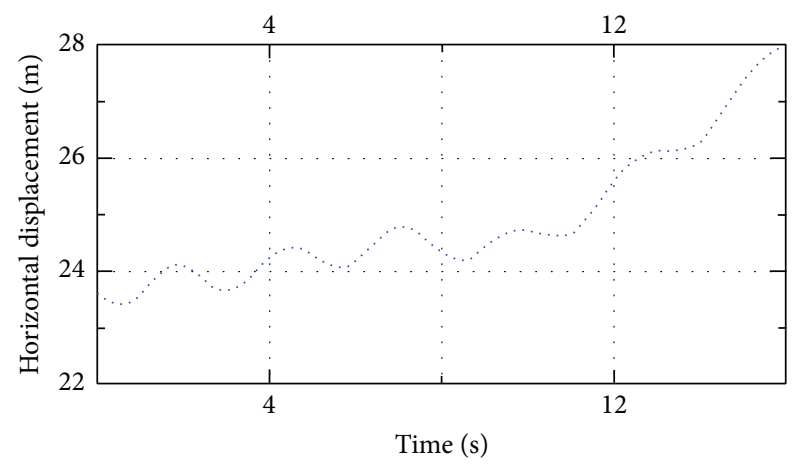

Horizontal displacement

Figure 6: The buoys movement. 


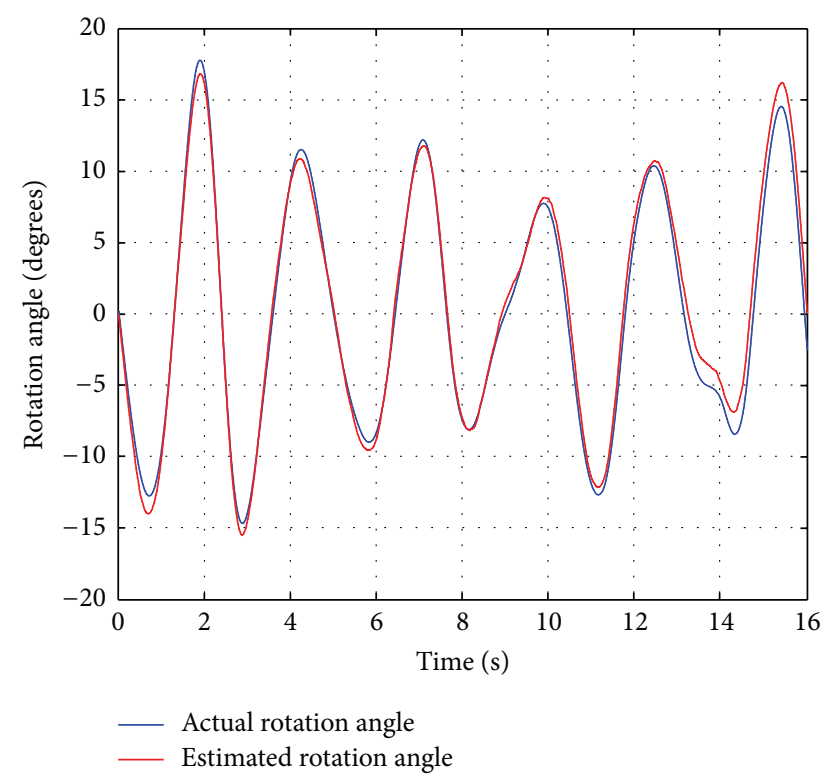

FIGURE 7: Typical results for actual and estimated angle $\phi$.

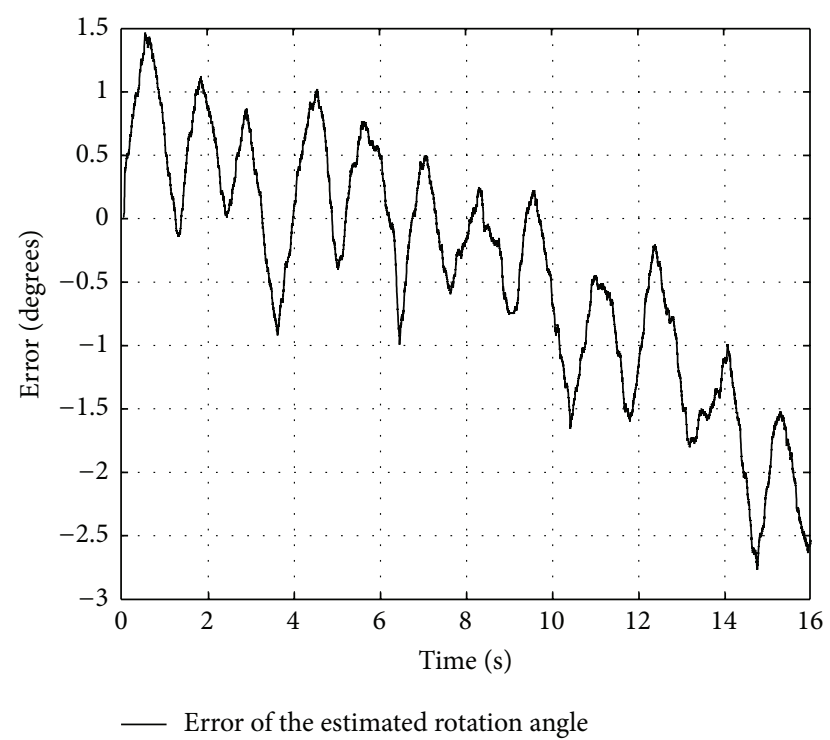

Figure 8: Typical results for error.

The results are shown in Figures 7 and 8. In Figure 7, the 2 traces represent the actual angles and the estimated angles, respectively. In Figure 8, the trace represents the calculated error in the estimated angles. From Figures 7 and 8, we find that the trace representing the filter estimated angles closely tracks the trace representing the actual angles, and the error is between -3 and 1.5 degrees. Figure 9 shows theoretical value and the simulative result of the power gain. Simulative results reveal that the beamforming technique provides significant power gains effectively. Figure 9 indicates that the antenna gain, ranging from 19.93 to $20 \mathrm{~dB}$, is obviously higher than that of antenna without beamforming.

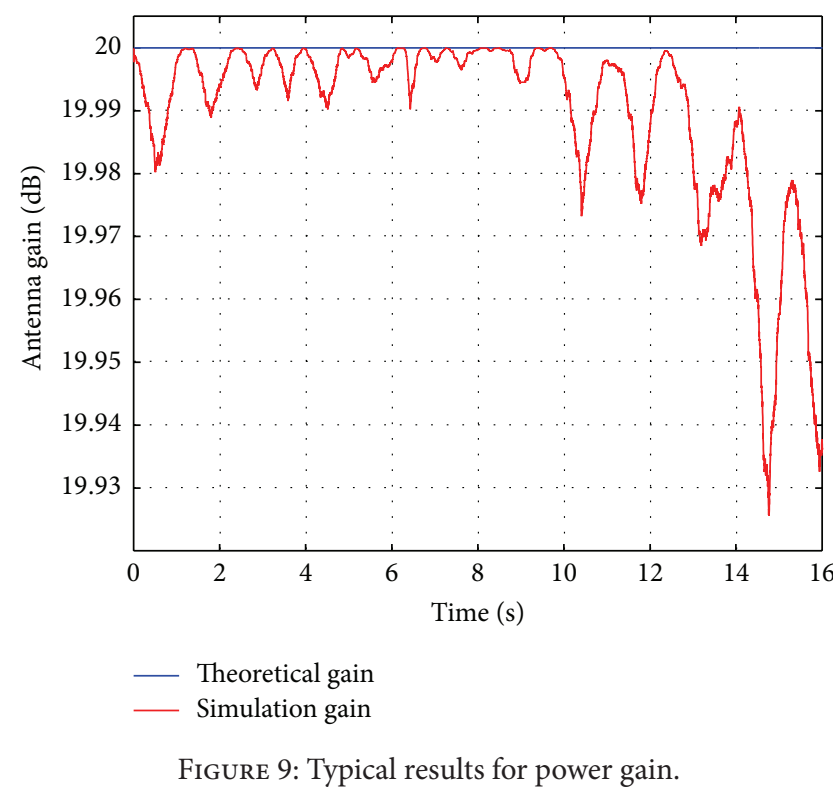

\section{Conclusion}

A novel beamforming scheme between buoys has been demonstrated in this paper. As was widely applied in the radar and sonar, the beamforming method was not used to extend the communication distance between buoys so far. Accelerator and gyroscope are installed on the buoy to track its movement. With the real-time attitude calculated by a certain scheme, the relative direction of the target node to the antenna array is obtained. Then a method is selected to perform beamforming. Finally, the scheme is tested and verified under the condition of regular waves. The results show that the beamforming effectively improves the antenna gain. Innovative aspect of the this paper is that this paper employs a method to get the relative direction of target node to the antenna array by installing an IMU on the buoy and then performs beamforming without channel estimation.

\section{Competing Interests}

The authors declare that they have no competing interests.

\section{Acknowledgments}

This work is in part supported by Natural Science Foundation of China under Grant no. 61371093.

\section{References}

[1] S. L. Wainfan, E. K. Wesel, M. S. Pavloff, and A. W. Wang, "Method and system for providing wideband communications to mobile users in a satellite-based network," US Patent: 6339707, 2002.

[2] C. Albaladejo, P. Sánchez, A. Iborra, F. Soto, J. A. López, and R. Torres, "Wireless sensor networks for oceanographic monitoring: a systematic review," Sensors, vol. 10, no. 7, pp. 6948-6968, 2010. 
[3] P. John, M. Supriya, and P. S. Pillai, "Cost effective sensor buoy for ocean environmental monitoring," in Proceedings of the MTS/IEEE Oceans Conference, pp. 1-5, Seattle, Wash, USA, 2010.

[4] B. D. Van Veen and K. M. Buckley, "Beamforming: a versatile approach to spatial filtering," IEEE ASSP Magazine, vol. 5, no. 2, pp. 4-24, 1988.

[5] J. Choi and J. R. W. Heath, "Interpolation based transmit beamforming for MIMO-OFDM with limited feedback," IEEE Transactions on Signal Processing, vol. 53, no. 11, pp. 4125-4135, 2005.

[6] Ö. Özdemir and M. Torlak, "Optimum feedback quantization in an opportunistic beamforming scheme," IEEE Transactions on Wireless Communications, vol. 9, no. 5, pp. 1584-1593, 2010.

[7] S. O. Madgwick, "An efficient orientation filter for inertial and inertial/magnetic sensor arrays," Report x-io, University of Bristol, Bristol, UK, 2010.

[8] T. Haynes, A Primer on Digital Beamforming, Spectrum Signal Processing, 1998.

[9] R. J. Mailloux, Phased Array Antenna Handbook, Artech House, Boston, Mass, USA, 2005.

[10] A. Innok, P. Uthansakul, and M. Uthansakul, "Angular beamforming technique for MIMO beamforming system," International Journal of Antennas and Propagation, vol. 2012, Article ID 638150, 10 pages, 2012.

[11] Z. Lin, X. Peng, and F. Chin, "Enhanced beamforming for $60 \mathrm{GHz}$ OFDM system with co-channel interference mitigation," in Proceedings of the IEEE International Conference on Ultra-Wideband (ICUWB '11), pp. 29-33, IEEE, Bologna, Italy, September 2011.

[12] R. Dean and R. Dalrymple, Water Wave Mechanics for Scientists and Engineers, vol. 2 of Advanced Series on Ocean, World Scientific, 1991. 


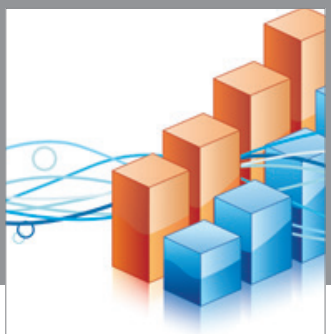

Advances in

Operations Research

vatem alat4

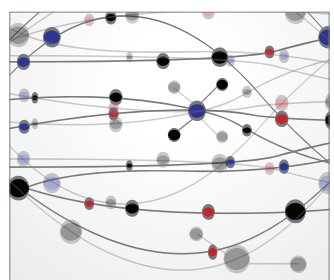

\section{The Scientific} World Journal
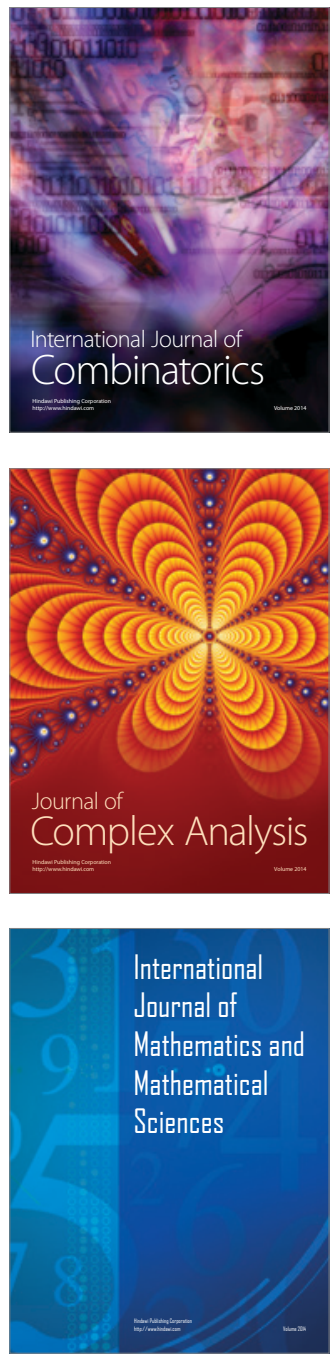
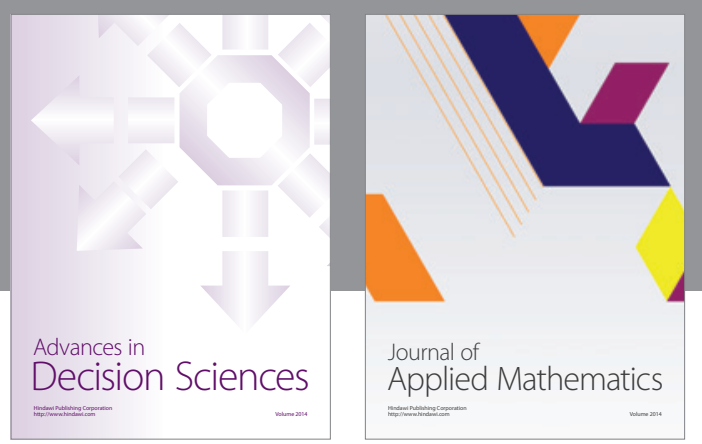

Algebra

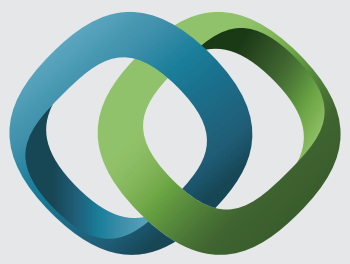

\section{Hindawi}

Submit your manuscripts at

http://www.hindawi.com
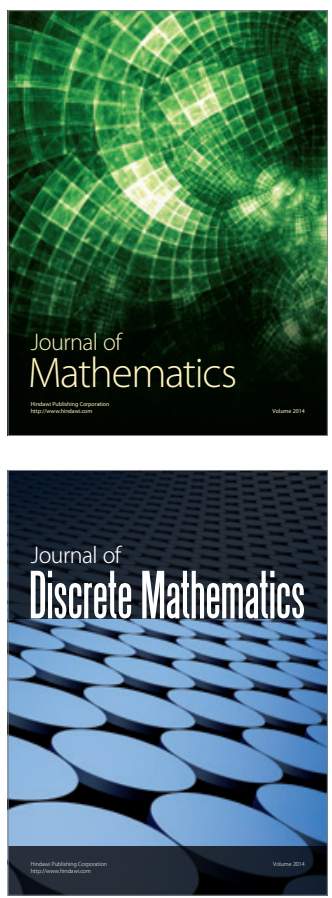

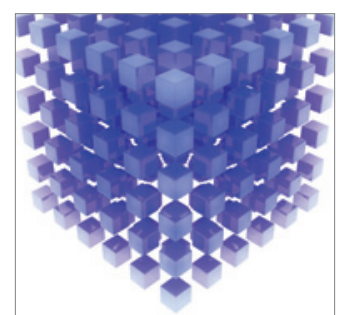

Mathematical Problems in Engineering
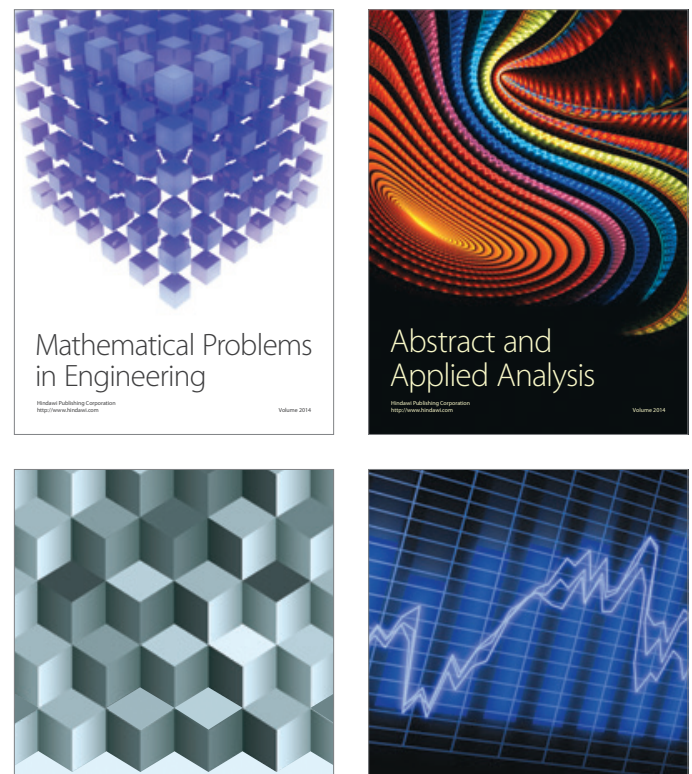

Journal of

Function Spaces

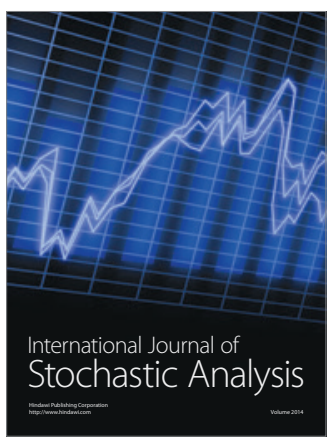

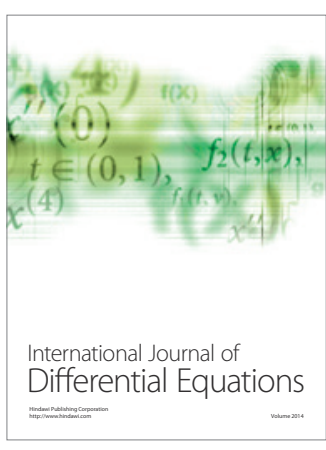
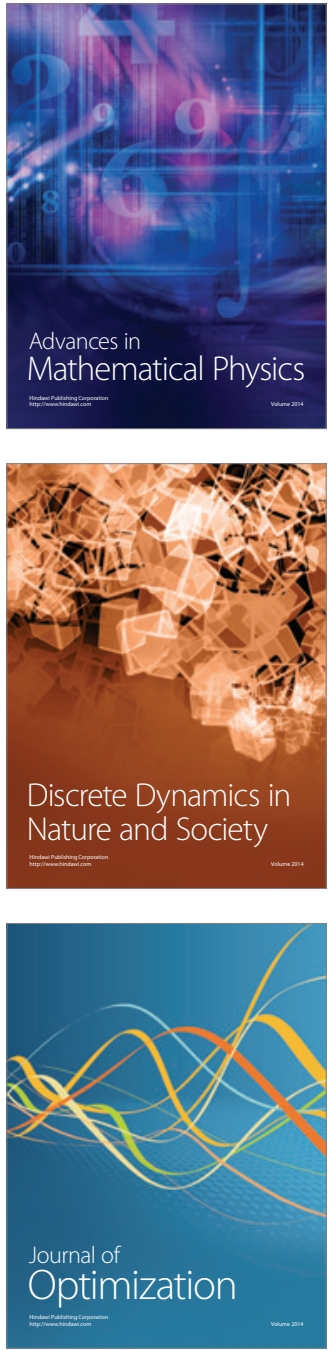